\title{
Minimal-invasive Techniken in der Unfallchirurgie
}

\author{
A. Schmidgen, O. Naumann, A. Wentzensen
}

\section{Zusammenfassung}

Minimal-invasive Verfahren nehmen durch verbesserte Operationstechnik und Perfektionierung der Implantate einen immer größer werdenden Raum in der Unfallchirurgie ein. Die reduzierte Weichteiltraumatisierung erlaubt eine schnellere funktionelle postoperative Rehabilitation mit kürzeren Krankenhausverweilzeiten. Auch der Trend zum ambulanten Operieren fordert minimale Invasivität. Bewährte Verfahren werden zum Teil modifiziert angewandt; es werden aber auch völlig neue Operationstechniken entwickelt. Die minimale Invasivität darf sich jedoch nicht nur auf die Hautinzision beziehen, sondern muss den gesamten operativen Zugangsweg berücksichtigen. Insbesondere bei der Versorgung von Gelenkverletzungen dürfen keine Kompromisse bei der Reposition und Stabilität eingegangen werden. Das computergesteuerte Operieren wird den Trend zum minimalinvasiven weiter vorantreiben.

\section{Einleitung}

Ein zielgerichteter, die Haut und umgebende Weichteile möglichst wenig schädigender Zugang zum Operationsgebiet ist seit jeher eine Grundforderung in allen operativen Disziplinen. Das operative Trauma durch den Chirurgen soll möglichst gering sein und keine zusätzliche Schädigung bedeuten. Durch sorgfältige OP-Planung für Zugangswege und Implantate ist die Idee des minimal-invasiven ein wesentlicher Bestandteil der Chirurgie. Verbesserte Techniken der intraoperativen Durchleuchtung, neue Implantatentwicklungen sowie das CT-gesteuerte und computernavigierte Operieren ebenso wie die zunehmende Perfektion in Arthroskopie und Endoskopie haben den Gedanken des Minimal-invasiven neu belebt. Zudem verspricht zumindest ein Teil der Techniken eine geringere postoperative Schmerzhaftigkeit, eine frühere Mobilisation und frühfunktionelle Nachbehandlung sowie kürzere Krankenhausbehandlungszeiten verbunden mit ebenfalls verkürzten Arbeitsunfähigkeitszeiten.

OP-JOURNAL 2000; 16: 138-142

(C) Georg Thieme Verlag Stuttgart . New York
Die Idealanforderungen an eine moderne minimal-invasive Technik sind folgende:

minimale, kosmetisch nicht störende Hautinzision

- minimale Weichteiltraumatisierung des operativen Zugangsweges

- optimale Reposition und Rekonstruktion

ausreichende Stabilisierung

minimale Implantate

frühfunktionelle Nachbehandlung.

\section{Perkutane Kirschner-Drähte}

Die perkutan eingebrachten Kirschnerdrähte sind ein altes und bewährtes minimal-invasives Verfahren. Es müssen folgende Prinzipien berücksichtigt werden: die Frakturgeometrie muss eine ausreichende Stabilisierung durch die Drähte zulassen; die Dicke des K-Drahtes sollte dem Knochen angepasst sein; parallel eingebrachte K-Drähte verhindern nicht das Zusammensintern der Fragmente; im Frakturspalt sich kreuzende K-Drähte ergeben keine Rotationsstabilität; der KDraht soll zum festen Sitz in der Gegenkortikalis verankert sein, hier jedoch die gegenüberliegenden Weichteile durch das spitze Drahtende nicht irritieren. Beim Einbringen des K-Drahtes ist auf Nerven und Gefäße zu achten; auch Sehnen sollten nicht durch die perkutanen Drähte blockiert sein.

Postoperativ verlangt der einheitlich KDraht eine sorgfältige, tägliche Pflege, wobei die Sekretverkrustungen entfernt und eine Desinfektion der Hauteintrittsstelle durchgeführt wird.

Die Compliance des Patienten ist somit bei der Auswahl des Verfahrens zu berücksichtigen.

Die K-Draht-Osteosynthese bedarf in aller Regel einer zusätzlichen Stabilisierung durch einen immobilisierenden Verband. Das räumliche Vorstellungsvermögen des Chirurgen ist entscheidend für die minimale Invasivität. Wiederholte Fehlbohrungen der Drähte schädigen nicht nur mechanisch die Weichteile und den $\mathrm{zu}$ stabilisierenden Knochen, sondern führen auch zu Hitzeschäden. Insbesondere in Gelenknähe können hier Gelenkkapsel- und -knorpelverletzungen das operative Ergebnis wesentlich beeinträchtigen.

Der handgelenksnahe Speichenbruch ohne Gelenkbeteiligung stellt nach wie vor eine Hauptindikation für die perkutane K-Drahtspickung dar (Abb.1).

Bei korrekter technischer Ausführung sind bei minimalem Aufwand gute Ergebnisse zu erwarten. Auch handgelenks-

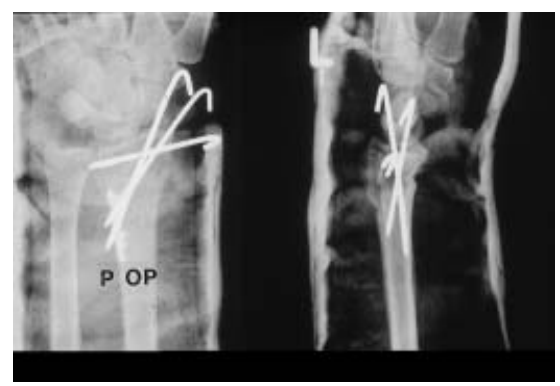

Abb.1 Durch 3 perkutan eingebrachte Kirschnerdrähte stabilisierte distale Radiusfraktur im Unterarmgipsverband. 
nahe, kindliche Unterarmfrakturen lassen sich sehr gut durch perkutan eingebrachte K-Drähte stabilisieren. Ebenso stellt die suprakondyläre Oberarmfraktur des Kindes mit noch offenen Epiphysenfugen eine sehr gute Indikation zur geschlossenen, gekreuzten K-Draht-Spikkung dar. Selbstverständlich ist auch hier die Lagebeziehung zum N. ulnaris zu beachten.

Zur Vermeidung von Achs- und Rotationsfehlern bedarf es der sorgfältigen Technik und suffizienten intraoperativen Röntgenkontrolle.

Die geschlossene Reposition und perkutane K-Draht-Stabilisierung der subkapitalen- und Humeruskopffraktur - insbesondere des alten Menschen - hat in letzter Zeit eine Renaissance erlebt. Es werden unterschiedliche Repositionstechniken mit vorgebogenen K-Drähten beschrieben $[1,2,3]$. Zur besseren Fixierung in der osteoporotischen, subchondralen Kopfkortikalis des Humerus werden auch Gewinde-Kirschner-Drähte eingebracht. Die Kombination der Reposition durch ein perkutan eingebrachtes Rasparatorium und der Stabilisierung durch K-Drähte ist ebenfalls möglich. Es ist darauf zu achten, dass die K-Drahtenden umgebogen werden, um eine Drahtwanderung zu verhindern.

Die frühfunktionelle Nachbehandlung ist jedoch nur sehr eingeschränkt möglich. I.d.R. wird für ca. 14 Tage eine Ruhigstellung im Gilchristverband erforderlich sein.

Bei der geschlossenen Reposition und Retention von dislozierten Mittelfuß- und Zehenfrakturen besteht nach wie vor eine gute Indikation zur K-Drahtstabilisierung. Ebenso haben sich die K-Drähte als minimal traumatisierendes Verfahren bei Handfrakturen bewährt. Sollte zur Stabilisierung von kindlichen Frakturen oder Epiphysenlösungen die Durchkreuzung einer Epiphysenfuge mit einem $\mathrm{K}$ Draht notwendig sein, ist darauf zu achten, dass maximal ein $1,6 \mathrm{~mm}$ dicker Draht verwendet wird, um das Risiko einer vorzeitigen, knöchernen Überbrükkung der Epiphysenfuge im Bohrbereich zu minimieren [4].

Die perkutane K-Drahtspickung stellt an die Erfahrung und das räumliche Vorstellungsvermögen des Chirurgen hohe Anforderungen.

\section{Intramedulläre Schienung}

Die Entwicklung dünner, elastischer Titannägel unterschiedlicher Konfiguration macht die minimal-invasive Stabilisierung von kindlichen Unterarm- und Oberschenkelschaftfrakturen im mittleren Drittel möglich [5]. Die Nägel lassen sich über eine kurze Hautinzision unter BV-Kontrolle rasch platzieren. Am Unterarm ist bei optimaler Lage der Nägel durch das Aufspreizen der Membrana interossea i.d.R. keine weitere äußere Immobilisation erforderlich und eine frühfunktionelle Behandlung möglich (Abb. 2).

Auch am Oberschenkel läßt sich durch das gekreuzte Einbringen von 2 Nägeln eine Rotationsstabilität erreichen. Die Weichteilinterposition als Repositionshindernis lässt sich ebenfalls rasch über

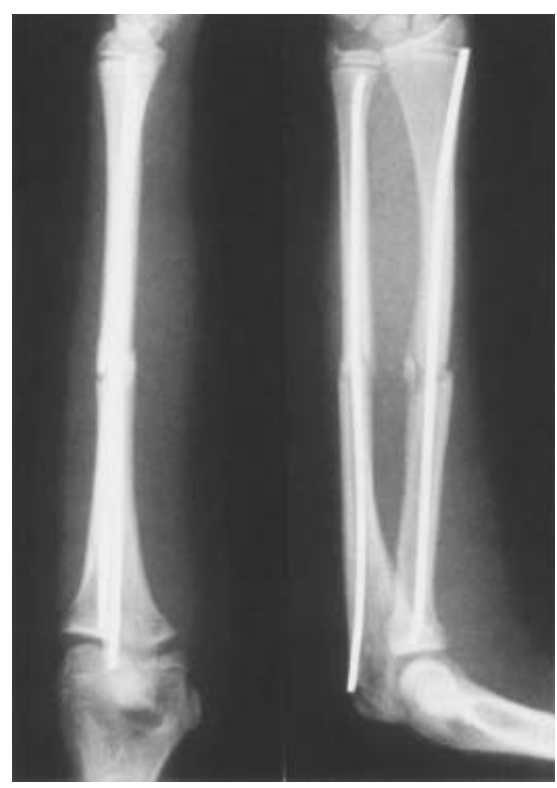

Abb. 2 Intramedulläre Schienung einer kindlichen Unterarmfraktur.

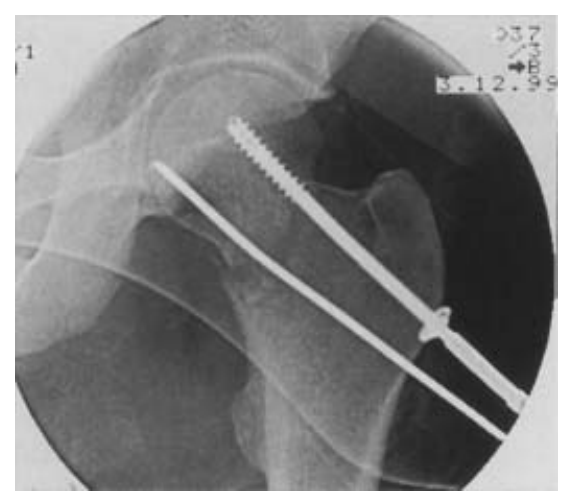

Abb. 3 Über Stichinzision eingebrachte Schenkelhalsschrauben. einen minimalen Zugang im Frakturbereich entfernen. Die Dicke des Nagels ist der Markhöhle anzupassen. Am Unterarm werden die Nägel von distal in den Radius und von proximal in die Ulna eingebracht. Hier muss eine Verletzung der Epiphysenfugen vermieden werden. Die beim Abkneifen der Nägel entstehenden scharfen Kanten sollten zur Vermeidung von Weichteilirritationen durch ein Plastikhütchen abgedeckt werden.

\section{Kanülierte Schrauben}

Bei diesem Verfahren wird eine Fraktur geschlossen oder durch einen minimalen Zugang reponiert und temporär durch einen perkutan eingebrachten dünnen Gewindedraht stabilisiert. Unter Bildwandler wird die korrekte Stellung der Fraktur sowie die optimale Lage des Drahtes kontrolliert und ggf. korrigiert, ohne dass ein nicht korrekter Bohrvorgang einen Knochensubstanzverlust hinterlässt. Bei dem korrekt liegenden Zieldraht kann dann die notwendige Schraubenlänge ausgemessen und der Bohrvorgang sicher geführt vorgenommen werden. Die Vorteile einer stabilen Schraubenosteosynthese lassen sich somit minimal-invasiv gezielt ausnutzen. Durch die Entwicklung von verschiedenen Schraubengrößen hat sich die Indikation zu dieser Osteosyntheseform sprunghaft erweitert.

Insbesondere durch die Möglichkeit der Korrektur des zunächst vorgelegten Zieldrahtes ist es ein sehr anwenderfreundliches Verfahren.

Durch eine minimale Stichinzision lassen sich mediale Schenkelhalsfrakturen durch 3 kanülierte Schrauben rasch und zuverlässig stabilisieren (Abb. 3). Monokondyläre Tibiakopffrakturen können durch dieses Verfahren - u.U. arthroskopisch kontrolliert, reponiert, über die perkutan eingebrachten Zieldrähte temporär fixiert und durch die Lochschrauben - im sponiösen Bereich mit Unterlegscheiben - stabilisiert werden. Ebenso besteht eine gute Indikation zur Versorgung von Talusfrakturen sowohl von ventral als auch von dorsal. Wir haben wiederholt Trümmerfrakturen des Pilon tibiale die primär mit einem gelenküberbrückenden Fixateur externe versorgt wurden, bei schwierigen Weichteilverhältnissen über minimale Stichinzisionen reponiert und über perkutan eingebrachte Kleinfragment-Lochschrauben osteosynthetisiert. 


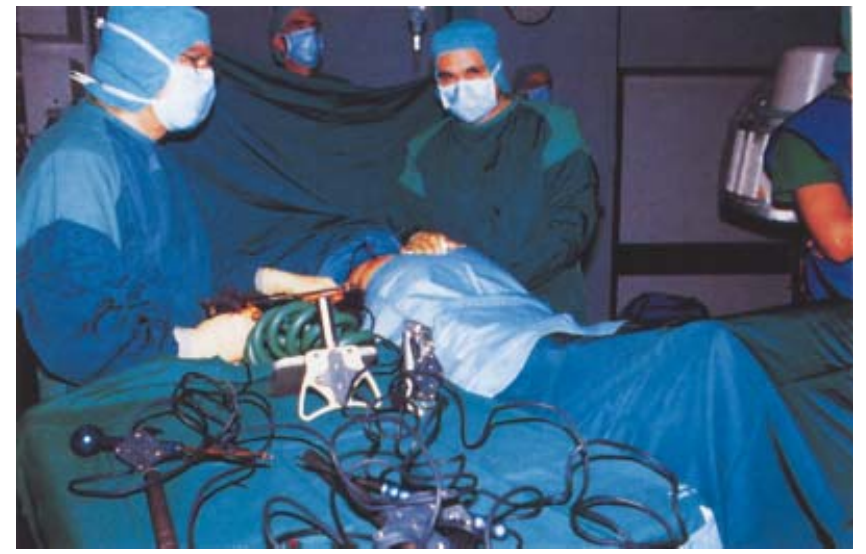

Abb. 4 Computernavigation.

Überall wo sich eine unverschobene oder durch eine minimale Inzision reponierbare Fraktur durch Schrauben stabil fixieren lässt, können Lochschrauben percutan eingebracht werden.

Hier sind der Fantasie der Chirurgen kaum Grenzen gesetzt. Allerdings dürfen im Gelenkbereich keine Kompromisse zu Lasten der Reposition und Stabilität gemacht werden. Von Resch wird eine Operationstechnik beschrieben, mit der auch 4-Segment-Frakturen des Humeruskopfes durch perkutane Manipulation reponiert und durch Lochschrauben und KDrähte mit gutem funktionellen Erfolg stabilisiert werden können $(6,7)$. Hierbei scheint es sich jedoch um ein schwieriges, dem sehr erfahrenen Operateur vorbehaltenes Verfahren zu handeln.

\section{Computernavigation}

Mit der Entwicklung des computergesteuerten Plazierens von Schrauben ist der Bohr- und Schraubvorgang 3-dimensional kontrollierbar, unabhängig von einer zum Teil schwierigen oder sogar unmöglichen intraoperativen BV-Kontrolle. Die Platzierung von perkutan eingebrachten Schrauben wird präzise und sicher (Abb.4).

Im klinischen Alltag ergeben sich z.Z. noch wiederholt Probleme bei der exakten Navigation. Des Weiteren ist eine Perfektionierung des Instrumentariums erforderlich. Bei dem rasanten technischen Fortschritt ist in naher Zukunft eine weite Verbreitung einer zuverlässigen und praktikablen Computernavigation zu erwarten, die die Sicherheit und Präzision von minimal-invasiven Techniken in bisher unvorstellbarem Maße verbessert. Ein sehr gutes Beispiel ist die computergesteuerte, perkutane IliosakralfugenVerschraubung. Nur durch dieses Verfah- ren ist die exakte, die Neuroforamina vermeidende Platzierung der Schrauben möglich.

\section{Eingeschobene Platte}

Tibiafrakturen im distalen Drittel, die eine intramedulläre Stabilisierung nicht mehr zulassen, können durch eine minimal-invasiv eingebrachte Platte versorgt werden. Hierzu wird über dem Innenknöchel eine 3-4cm lange Hautinzision angelegt. Über diese kann dann zwischen dem Periost der medialen Schienbeinfläche und dem Subkutangewebe eine anatomisch vorgebogene $4,5 \mathrm{~mm}$ schmale LCDC-Platte eingeschoben werden. Die Schrauben werden unter Bildwandlerkontrolle durch perkutane Stichinzisionen direkt über den Plattenlöchern eingebracht. Auch die Platzierung einer Zugschraube durch ein Plattenloch ist möglich. Die korrekte Achsen- und Rotationseinstellung verlangt jedoch ein gewisses Maß an Erfahrung (Abb.5). Selbst periprothetische Oberschenkelfrakturen können durch eine in dieser Technik eingeschobene Winkelplatte stabilisiert werden.

\section{LISS (Less invasive stabilisation system)}

Dieses System bietet die Möglichkeit, über eine minimale Hautinzision distale Femurfrakturen und proximale Tibiafrakturen mit einer Platte winkelstabil zu stabilisieren. Auch schwierige, gelenknahe Frakturen lassen sich hierdurch versorgen. Über ein Zielgerät werden die sich winkelstabil in der Platte verankernden Schrauben eingebracht (Abb.6).

Diese Platte ist als „Fixateur-Interne“ konzipiert. Sie liegt dem Knochen nicht direkt auf, sondern hat einen kleinen Abstand, so dass hier die periostale Durchblutung nicht gestört wird. Andererseits

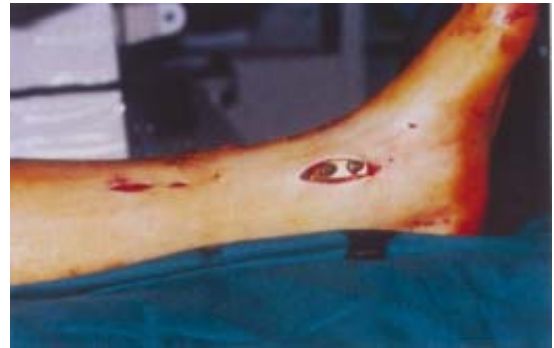

Abb. 5 Kurze Hautinzision für eine eingeschobene LCDC-Platte bei distaler Tibiafraktur.

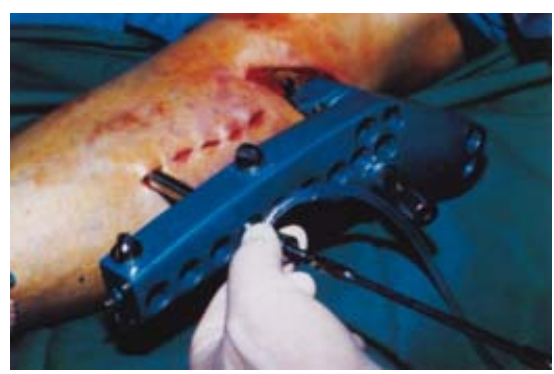

Abb. 6 LISS-Platte mit Zielbügel.

lässt sich jedoch die Fraktur nicht an der Platte reponieren. Die Fraktur sollte deswegen vor Einbringen der Platte anatomiegerecht stehen oder sogar durch einen temporär angebrachten Fixateur externe in der korrekten Achsausrichtung stabilisiert sein. Bei Gelenkflächen einbeziehenden Frakturen ist u.U. die zusätzliche Platzierung von Zugschrauben erforderlich.

\section{Fixateur externe}

Dieses Verfahren darf als minimal-invasives Verfahren nicht unerwähnt bleiben. Er erfüllt in nahezu idealer Weise die Anforderungen für ein minimal-invasives System, und ist in der Lage, auch schwierigste Frakturformen und Weichteilsituationen zu beherrschen. Weiterhin bietet der Fixateur als Bewegungsfixateur an Hand- und Ellbogengelenk die Möglichkeit der frühfunktionellen Behandlung bei zuverlässiger Stabilisierung (Abb. 7). Auch das weite Feld der Beinachsenkorrektur und Extremitätenverlängerung durch den Fixateur externe ist im eigentlichen Sinne ein minimal-invasives Verfahren [8]. Computerunterstützt ist mit dem Hexapod-Fixateur eine „Punktlandung" möglich.

\section{Fixateur interne}

Verheyden und Josten haben ein Verfahren der perkutanen Implantation eines Wirbelsäulen-Fixateur-interne beschrie- 


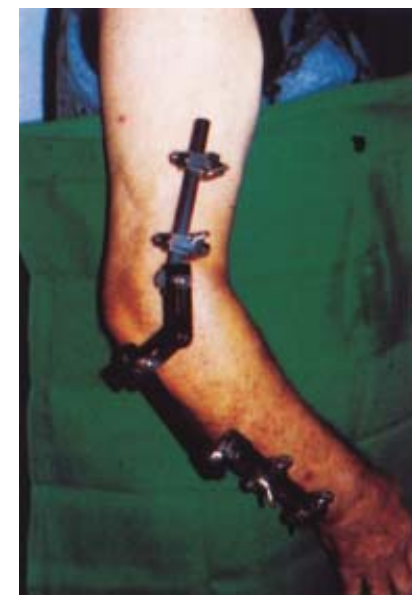

Abb. 7 Bewegungsfixateur am Ellenbogengelenk.

ben [9]. Durch 4 je $3 \mathrm{~cm}$ lange Hautinzisionen werden perkutan die dorsalen Eintrittspunkte für die Pedikel aufgesucht und die Schanz-Schrauben platziert. Unter Zuhilfenahme der Computernavigation kann hier ohne vermehrte Strahlenbelastung die Zuverlässigkeit und Sicherheit des Aufsuchens der Pedikel noch verbessert werden.

\section{Unaufgebohrte Nägel}

Die Stabilisierung von Schaftfrakturen langer Röhrenknochen durch einen intramedullären Kraftträger der weit von der Fraktur durch eine minimale Hautinzision eingebracht wird, erfüllt ebenfalls die Voraussetzungen für ein minimal-invasives Verfahren. Die zusätzliche Operationstraumatisierung ist sehr gering bei einer hervorragenden mechanischen Stabilisierung der Fraktur unter Erhaltung des Frakturhämatoms. Mit der Modifikation des unaufgebohrten Nagels wird die Invasivität weiter vermindert, indem die hinlänglich beschriebene Schädigung der Markhöhle durch den Aufbohrvorgang entfällt. Durch eine statische und dynamische Verriegelung ist eine zusätzliche Fixierung der Knochenlänge und Rotation möglich. Unaufgebohrte Nägel sind als unaufgebohrter Femurnagel (UFN), proximaler Femurnagel (PFN), distaler Femurnagel (DFN), unaufgebohrter Tibianagel (UTN) und unaufgebohrter Humerusnagel (UHN) (Abb. 8) im Einsatz. Der UHN bietet den Vorteil, dass er über eine ca. 3$4 \mathrm{~cm}$ lange Inzision proximal des Olekranons fern des N. radialis eingebracht werden kann. Die proximalen Verriegelungsschrauben dieses Nagels liegen jedoch im Bereich des N. axillaris. Eine Nervenschädigung ist durch eine sorgfältige Präparation zu vermeiden [10,11].
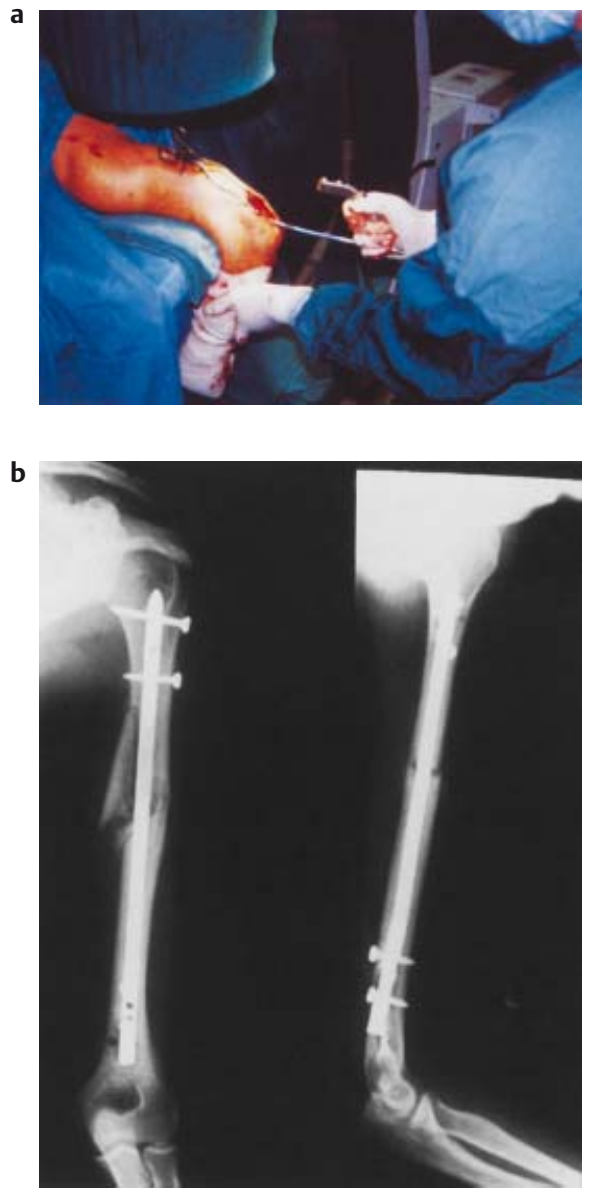

Abb. 8 Unaufgebohrter Humerusnagel

\section{Arthroskopie}

Das moderne arthroskopische Instrumentarium hat den minimal-invasiven Zugang zu fast sämtlichen Gelenken des menschlichen Körpers ermöglicht [12]. Durch das Einbringen des Arthroskops und des Instrumentariums über Stichinzisionen wird die Gelenkkapsel und damit die Propriozeption des Gelenkes nur minimal gestört, was die früh-funktionelle Nachbehandlung und Rehabilitation wesentlich erleichtert (Abb.9). Ein differenziertes System mechanischer Instrumente, die Entwicklung der Elektround Lasertherapie hat die Meniskusund Bandchirurgie revolutioniert. Insbesondere bei der Kreuzbandchirurgie entwickelt sich die Kombination von Arthroskopie und Computernavigation. Auch die Behandlung von subchondralen Knochenzysten oder der Osteochondrosis dissecans bietet eine Möglichkeit der kombinierten Anwendung von Arthroskopie und Computernavigation.

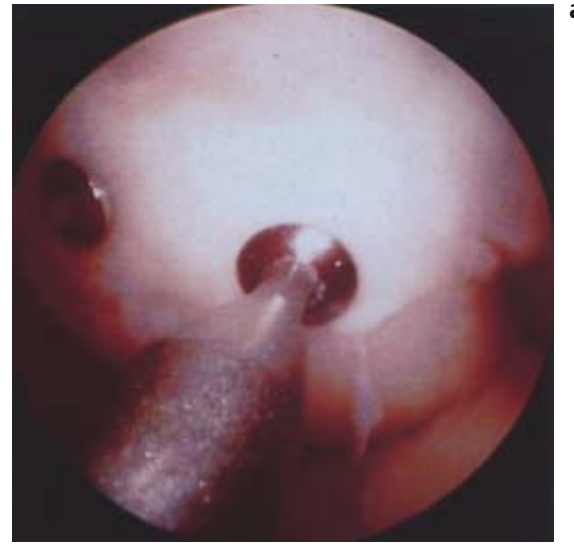

Abb.9a Arthroskopische Schraubenentfernung nach Refixation einer Knorpel-Knochenabscherung an der lateralen Fermurkondyle.

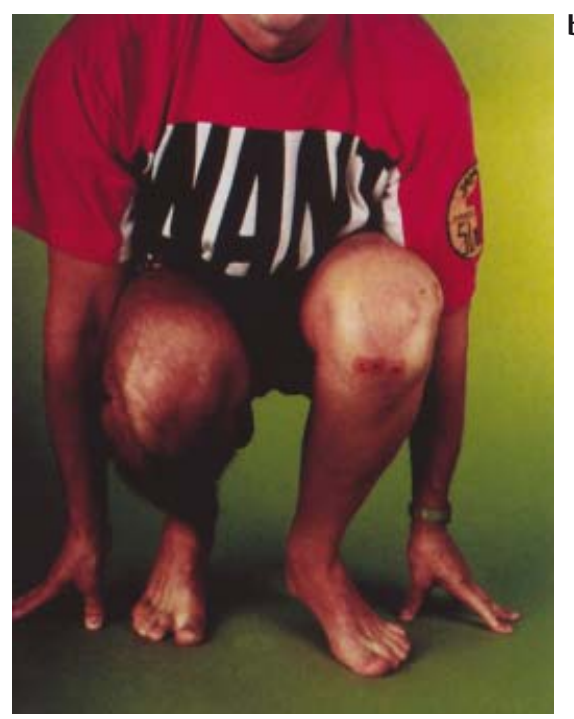

Abb.9b Postoperative, frühfunktionelle Nachbehandlung nach arthroskopischer Schraubenentfernung.

\section{Thorakoskopische/Endoskopische Wirbelsäulenchirurgie}

Die Entwicklung spezieller Instrumente und Implantate hat es ermöglicht, durch minimale Inzisionen ventrale Stabilisierungen von Wirbelfrakturen vorzunehmen [13]. Die bisher üblichen, ausgedehnten, im postoperativen Verlauf schmerzhaften, komplikationsträchtigen und die Rehabilitation verzögernden $\mathrm{Zu}$ gänge entfallen. Die Vorteile der minimalen Invasivität werden jedoch gerade hier durch eine längere Operationszeit und eine flache Lernkurve des Operateurs erkauft. Diese Verfahren werden aus diesem Grunde wahrscheinlich auch in $\mathrm{Zu}$ kunft nur in spezialisierten Zentren routinemäßig eingesetzt werden können. 

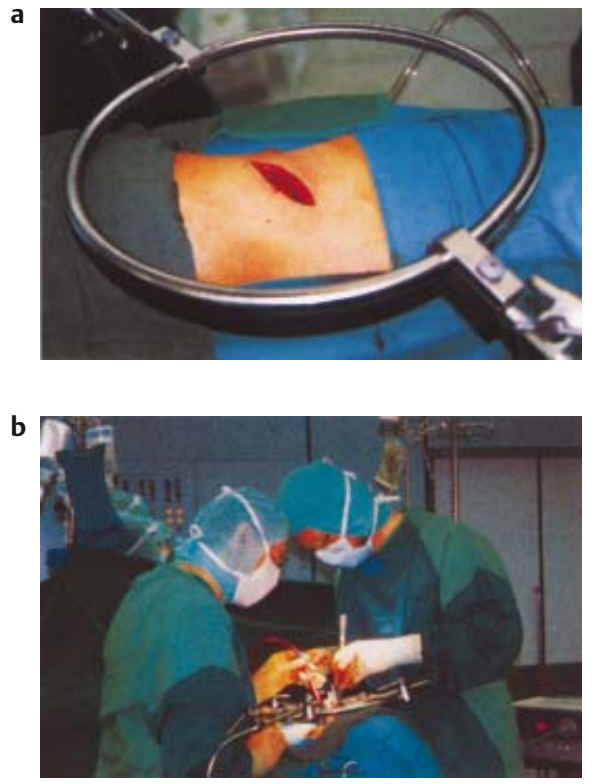

Abb.10au.b Ventrale Spondylodese über minimalen Zugang mit Hilfe des SynFrame.

Einen Kompromiss bietet der jetzt im Handel befindliche SynFrame: ein Hakenhaltesystem, das an einem am OPTisch befestigten Ring verstellbar montiert werden kann. Durch dieses System können auch ventrale Stabilisierungen der LWS, der unteren und mittleren BWS über 4-6 cm lange Hautinzisionen durchgeführt werden. Die Vorteile der minimalen Invasivität des endoskopischen Verfahrens werden mit der direkten Zugänglichkeit des offenen Verfahrens kombiniert (Abb.10a u.b).

\section{Perkutane Techniken der Achillessehne}

In jüngster Vergangenheit sind unterschiedliche Techniken der perkutanen Achillessehnennaht beschrieben worden $[14,15]$. Durch kleine Stichinzisionen werden Nähte durch die Achillessehne eingebracht und unter Wiederherstellung der Sehnenspannung subkutan geknotet. Hierbei bleibt das für die Sehnenernährung wichtige Peritendineum unangetastet. Pfeil beschreibt ein perkutanes Achillessehnen-Verlängerungsverfahren. Durch minimale Stichinzisionen werden mit einem Tenotom senkrecht zur Achillessehne fersennah, am proximalen Ende und zuletzt an der Gegenseite im mittleren Drittel unter leichter Anspannung Durchtrennungen bis etwa zur Sehnenmitte durchgeführt. Die Sehnenanteile sollen bei passiver Extension im Sprunggelenk aneinander vorbeigleiten, und die Verlängerung durch das Ausmaß der Extension dosierbar sein.

\section{Perkutane Songiosiagewinnung}

Durch die klinische Erfahrung ist hinreichend bekannt, dass die Patienten nach Spongiosaentnahme vom Beckenkamm hier häufig über stärkere Schmerzen klagen als im eigentlichen Operationsgebiet. Außerdem treten hier gehäuft Komplikationen in Form von Hämatomen und Schäden des N. cutaneus femoris lateralis auf. Von Jerosch wurde ein minimal-invasives Verfahren beschrieben, das die Spongiosaentnahme aus dem Beckenkamm durch eine Hohlnadel erlaubt [16]. Mehrere, bis zu $8 \mathrm{~cm}$ lange Spongiosazylinder können gewonnen werden. Die postoperativen Beschwerden und Komplikationen sollen deutlich geringer sein als bei der offenen Entnahme.

Die oben beschriebenen minimal-invasiven Techniken bedeuten sicher einen Fortschritt in der Patientenversorgung, wenn sie von erfahrenen Chirurgen unter Beachtung der allgemeinen Regeln der Unfallchirurgie eingesetzt werden.

Die minimale Hautinzision darf nicht durch eine maximale Gewebstraumatisierung unter der Haut erkauft werden. Ebenso dürfen keine Kompromisse in der Reposition und Sorgfalt der Gelenkflächenrekonstruktion eingegangen werden. Die Anwendung dieser Techniken verlangt ein gutes räumliches Vorstellungsvermögen und eine ausreichende operative Erfahrung.

Sicher wird die Entwicklung der minimal-invasiven Techniken durch den breiter und perfekter werdenden Einsatz des computergesteuerten Navigierens einen weiteren Schub erhalten.

\section{Literatur}

${ }^{1}$ Jaberg H, Warner JP, Jakob RP. Percutaneous stabilization of unstable fractures of the humerus J Bone Joint Surg 1992; 74 A: 508-515

${ }^{2}$ Lahm A, Roesgen M. Minimalosteosynthese mit Drahtcerclagen bei dislozierten Humeruskopffrakturen. Akt Traumatol 1996; 26: $22-28$

${ }^{3}$ Lill H, Giers R, Schmidt A, Echtermeyer V. Die subcapitale Humerusfraktur. Operative Technik mit einer modifizierten KirschnerDrahttechnik. Chir Praxis 1996; 50: 427 - 438

${ }^{4}$ Laer von L. Frakturen und Luxationen im Wachstumsalter. Thieme Verlag, 3. Aufl., Stuttgart, New York 1996

${ }^{5}$ Dietz HG, Schmittenbecher PP, Illing P. Intramedulläre Osteosynthese im Wachstumsalter. Urban \& Schwarzenberg, München, Wien, Baltimore 1997

${ }^{6}$ Povacz P, Resch H. Osteosynthese bei proximalen Humerusfrakturen. Ther Umsch 1998; 55: $192-196$

${ }^{7}$ Resch H, Povacz H, Fröhlich R, Wambacher M. Percutaneous fixation of three- and four-part fractures of the proximal humerus. J Bone Joint Surg 1997; 79 B: 295-300

${ }^{8}$ Strecker W, Keppler P, Kinzl L. Posttraumatische Beindeformitäten. Springer, Berlin, Heidelberg 1997

${ }^{9}$ Verheyden P, Josten C. Percutaner Wirbelsäulen-Fixateur interne. In: Pfeil J, Siebert W, Janousek A, Josten C. Minimal-invasive Verfahren in der Orthopädie und Traumatologie. Springer, Berlin, Heidelberg 2000; S. 30-33

${ }^{10}$ Krettek C, Schulte-Eistrup S, Schandelmaier $\mathrm{P}$, Rudolf J, Tscherne H. Osteosynthese von Femurschaftfrakturen mit dem unaufgebohrten AO-Femurnagel (UFN) - Operative Technik u. erste klein. Ergebnisse mit der Standardverriegelung. Unfallchirurg 1997

${ }^{11}$ Verheyden P, Streidt A, Lill H, Weise K, Josten C. Der unaufgebohrte Humerusnagel - Indikationen, Technik u. klin. Erfahrungen. Akt Traumatologie 1998; 6: $251-257$

${ }^{12}$ Kohn D. Diagnostische und operative Arthroskopie großer Gelenke. Thieme Verlag Stuttgart, New York 1997

${ }^{13}$ Bühren V, Beisse R, Potulski M. Minimal-invasive ventrale Spondylodesen bei Verletzungen der Brust- und Lendenwirbelsäule. Chirurg 1997; 68: 1076 - 1084

${ }^{14}$ Ma GW, Griffith TG. Percutaneous repair of acute closed ruptured achilles tendon: a new technique. Clin Orthop 1977; 128: 247-255

${ }^{15}$ Buchgraber A, Possler HH. Percutaneous repair of Achilles tendon rupture. Immobilisation Versus functional postoperative treatment. Clin Orthop 1997; 341: 113-122

${ }^{16}$ Jerosch J, Castro WHM, Steinbeck J. Perkutane Spongiosaplastik für minimal invasive Eingriffe. Orthop Prax 1995; 31: 632-635

\section{Dr. med. A. Schmidgen \\ Oberarzt}

Dr. med. 0. Naumann

Assistenzarzt

Prof. Dr. med. A. Wentzensen

Ärztlicher Direktor

BG-Unfallklinik Ludwigshafen

Ludwig-Guttmann-Str. 13

67071 Ludwigshafen 